\title{
Artificial Intelligence Chatbot Behavior Change Model for Designing Artificial Intelligence Chatbots to Promote Physical Activity and a Healthy Diet: Viewpoint
}

Jingwen Zhang ${ }^{1,2}, \mathrm{PhD}$; Yoo Jung $\mathrm{Oh}^{1}, \mathrm{MA}$; Patrick Lange ${ }^{3}$, MSc; Zhou Yu ${ }^{3}, \mathrm{PhD}$; Yoshimi Fukuoka ${ }^{4}, \mathrm{PhD}, \mathrm{RN}$, FAAN

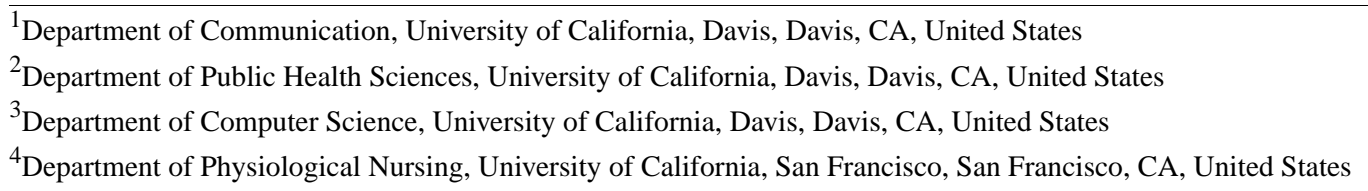

Corresponding Author:

Jingwen Zhang, $\mathrm{PhD}$

Department of Communication

University of California, Davis

One Shields Avenue

Davis, CA, 95616

United States

Phone: 15307541472

Email: jwzzhang@ucdavis.edu

\section{Abstract}

Background: Chatbots empowered by artificial intelligence (AI) can increasingly engage in natural conversations and build relationships with users. Applying AI chatbots to lifestyle modification programs is one of the promising areas to develop cost-effective and feasible behavior interventions to promote physical activity and a healthy diet.

Objective: The purposes of this perspective paper are to present a brief literature review of chatbot use in promoting physical activity and a healthy diet, describe the AI chatbot behavior change model our research team developed based on extensive interdisciplinary research, and discuss ethical principles and considerations.

Methods: We conducted a preliminary search of studies reporting chatbots for improving physical activity and/or diet in four databases in July 2020. We summarized the characteristics of the chatbot studies and reviewed recent developments in human-AI communication research and innovations in natural language processing. Based on the identified gaps and opportunities, as well as our own clinical and research experience and findings, we propose an AI chatbot behavior change model.

Results: Our review found a lack of understanding around theoretical guidance and practical recommendations on designing AI chatbots for lifestyle modification programs. The proposed AI chatbot behavior change model consists of the following four components to provide such guidance: (1) designing chatbot characteristics and understanding user background; (2) building relational capacity; (3) building persuasive conversational capacity; and (4) evaluating mechanisms and outcomes. The rationale and evidence supporting the design and evaluation choices for this model are presented in this paper.

Conclusions: As AI chatbots become increasingly integrated into various digital communications, our proposed theoretical framework is the first step to conceptualize the scope of utilization in health behavior change domains and to synthesize all possible dimensions of chatbot features to inform intervention design and evaluation. There is a need for more interdisciplinary work to continue developing AI techniques to improve a chatbot's relational and persuasive capacities to change physical activity and diet behaviors with strong ethical principles.

(J Med Internet Res 2020;22(9):e22845) doi: 10.2196/22845

\section{KEYWORDS}

chatbot; conversational agent; artificial intelligence; physical activity; diet; intervention; behavior change; natural language processing; communication 


\section{Introduction}

\section{Background}

Physical inactivity and an unhealthy diet continue to be some of the leading risk factors for noncommunicable diseases (NCDs), such as cardiovascular disease, diabetes, and obesity $[1,2]$, and death worldwide [3]. NCDs account for seven out of 10 deaths worldwide [3] and pose a substantial economic burden [4]. The prevalence of physical inactivity and an unhealthy diet varies considerably within and across countries. The United States is one of the countries experiencing a rapid rise in these risks. Nearly $80 \%$ of American adults do not meet the guidelines for both aerobic and muscle-strengthening activities [5], and the prevalence of overweight or obesity reached $71.6 \%$ in 2016 [6]. Therefore, developing cost-effective and feasible lifestyle interventions is urgently needed to reduce the prevalence [7].

Lifestyle modification programs have consistently evolved with emerging digital and communication technologies [8-13]. In the past two decades, there has been a large number of published studies using internet and mobile-based behavior interventions to support the effectiveness of using digital technologies to deliver intervention materials to diverse populations $[8,14]$. In recent years, the use of artificial intelligence (AI) and associated computational techniques has become the new frontier in expanding the landscape of health care and interventions [15].

\section{Definition and Applications of an AI Chatbot}

AI chatbots, also called conversational agents, employ dialog systems to enable natural language conversations with users by means of speech, text, or both [16]. Conceptually, the core technical capacity of AI chatbots is different from that of embodied virtual conversational agents or avatars that emphasize on synthesizing multimodal signals (eg, images, videos, and sounds) to simulate human face-to-face communication. In this paper, we focused on developing the AI chatbot's core feature of natural language conversation to facilitate more flexible information exchange between humans and the chatbot. The conversational capacity can range from constrained conversation (ie, users can only respond by selecting predefined conversational lines) to unconstrained conversation (ie, users can respond freely by inputting natural language conversational lines).

AI chatbots can be deployed in the form of mobile apps on smartphones, thus making programs available 24/7. AI chatbots have been rapidly transforming multiple fields, including business [17], governance [18], education [19], and health care $[16,20]$. As the top platforms supporting chatbot development, Amazon Alexa had more than 100,000 programs and Facebook Messenger had more than 300,000 active chatbots as of 2019, many of which are for health care and wellbeing. For instance, in April 2020, the World Health Organization launched a chatbot on Facebook Messenger to combat misinformation and to offer instant and accurate information about COVID-19 [21].

As chatbots increasingly become a convenient digital communication channel, they open up many opportunities for delivering personalized behavior change programs for disease prevention and health promotion on a large scale. Beyond connectivity and feasibility, the advantages of AI chatbot programs lie essentially in the computational power to develop and deliver personalized interventions [22-24]. Such interventions have the potential to overcome several limitations in the traditional paradigm of nonpersonalized interventions, as they are designed based on understanding individual characteristics and behavior trajectories and can incrementally adapt intervention strategies based on contextual conditions and personal cognitive and emotional states over time. In other words, chatbot technologies have the potential to "understand" individuals through natural human conversations, persuade individuals to change, and build sustaining supportive relationships for maintaining healthy behaviors.

\section{AI Chatbots for Health Care and Lifestyle Modification Programs}

Chatbots for promoting physical activity and a healthy diet are designed to achieve behavior change goals, such as walking for certain times and/or distances and following healthy meal plans [25-29]. Although no systematic review of chatbots for lifestyle modification programs has been published, there are several reviews on chatbots covering health care issues ranging from mental health support and smoking cessation to disease diagnosis $[16,30]$. Owing to the different natures of targeted behaviors, some chatbots were mainly designed to provide information and knowledge [31], whereas others were developed based on established mental health intervention programs such as cognitive behavioral therapy [32]. One relevant review [33] focused on discussing the development of embodied conversational agents for a healthy lifestyle, and pointed out that the interpretation and application of behavior change theories were usually not reported.

Most previous chatbot research relied on either finite-state (ie, dialog consisting of a sequence of predetermined steps or states) or frame-based systems (ie, dialog is not predetermined but dependent on the content of the user's input and the information that the system has to elicit) [34-36]. Such systems are restrained in their ability to allow free conversations, primarily due to the lack of large training data sets on human-to-human conversations in domains involving behavior changes.

The recent success of large pretrained language models, such as Bidirectional Encoder Representations from Transformers (BERT) developed by Google [37] and Generative Pre-Training-2 (GPT2) developed by Open AI [38], provides promising opportunities to incorporate language priors to down-stream natural language processing (NLP) tasks. For instance, several papers have shown that pretrained models can be tailored for task-oriented dialog generation, such as for conversations about restaurant recommendations and donation persuasion $[39,40]$. BERT and GPT2 are giant neural network models trained with large text data sets using self-supervised task objectives, such as recovering masked tokens and predicting the next word. As these models operate on representation space and do not have access to symbolic common-sense information, they produce outputs that are difficult for humans to interpret and can make errors that violate common senses in specific domains. One general direction to advance this field is to build systems that incorporate pretrained models to facilitate building 
dialogs that are specific for communicating and persuading users to adopt regular physical activity and a healthy diet.

To advance the science of developing effective and ethical AI chatbots for health behavior changes, especially within the context of improving physical activity and healthy eating behaviors, we provide a theoretical perspective and a model to guide the development and evaluation of AI chatbots for behavior changes. The aims of this perspective paper are threefold as follows: (1) to briefly summarize the current state of applications of AI chatbots in promoting physical activity and a healthy diet; (2) to propose the AI chatbot behavior change model developed by our research team; and (3) to address ethical considerations and principles.

\section{Methods}

\section{Preliminary Review of AI Chatbot-Based Physical Activity and Diet Interventions}

To provide a background of the current state of chatbot-based behavior interventions for physical activity and diet, we conducted a rapid preliminary literature review using four electronic databases (PubMed, EMBASE, Web of Science, and ACM Digital Library) on August 24, 2020. We used a combination of keywords to identify peer-reviewed studies related to AI chatbots for physical activity or diet (ie, ["chatbot" OR "conversational agent" OR "conversational system" OR "dialog system" OR "dialogue system" OR "relational agent"] AND ["physical activity" OR "exercise" OR "diet" OR "nutrition"]). We included only full-length articles that reported chatbot-based physical activity or diet interventions and were written in English. One researcher initially screened study titles and abstracts to determine eligibility for inclusion. Thereafter, two researchers reviewed the full texts of the included studies to further determine their relevance and coded study features. The two researchers discussed their disagreements throughout the coding process and agreed upon the final results.

In total, the search returned 108 articles from the four databases, with 15 published articles in 2020, 26 in 2019, 15 in 2018, 14 in 2017, five in 2016, and the remaining 33 from 2015 or before. After the screening, 101 (93.5\%) articles were excluded for the following reasons: commentary or opinion pieces, scoping reviews, or empirical studies that addressed health domains other than physical activity and diet (eg, chatbots assisting diagnostic tasks or offering mental health interventions or treatment).

\section{Characteristics of AI Chatbot Interventions}

We identified seven articles reporting six unique chatbots to increase physical activity and/or adoption of a healthy diet (Multimedia Appendix 1). Two papers reported on the same chatbot called Assistant to Lift your Level of activitY (Ally). One protocol [41] described the study design and one reported the actual optimization randomized controlled trial (RCT) $(n=274)$ [42] for evaluating the effects of Ally in helping users to reach personalized daily step goals. The results showed that the intervention component of daily cash incentives delivered by Ally increased step-goal achievement. However, 30\% of participants stopped using the app over the course of the study, presenting a challenge for the chatbot's ability to engage participants. In contrast, another study reported the results of an $\operatorname{RCT}(n=106)$ [43] to evaluate the Healthy Lifestyle Coaching chatbot. The findings demonstrated that this chatbot was effective in increasing physical activity after 12 weeks of the intervention among office workers. The remaining four studies employed pretest-posttest designs. One feasibility study $(n=23)$ [44] tested Tess, a behavioral coaching chatbot, in assisting adolescent patients to cope with weight management and prediabetes symptoms. Patients actively engaged with the chatbot, reported experiencing positive progress toward their goals, and deemed the chatbot helpful. One proof-of-concept study [45] reported on the Paola chatbot, which provided educational messages on physical activity and diet, weekly check-ins, and answers to user questions. The results showed that participants reported relevant weight loss and improved diet. Another validation study [46] reported on the CoachAI chatbot, which provided social and tailored health coaching support, and found this chatbot to be effective, especially among users with high engagement levels. Lastly, a chatbot named Reflection Companion delivered daily adaptive mini-dialogs and activity graphs to promote self-reflections. The conversations successfully triggered self-reflections that led to increased motivation, empowerment, and adoption of physical activity behaviors (eg, walking to a grocery store instead of taking a car) [26].

The above-reviewed chatbots showed preliminary evidence supporting the efficacy of using chatbots to deliver physical activity and diet interventions. It is worth noting that four out of seven $(57.1 \%)$ studies reported chatbots as the only intervention used to deliver behavior change strategies $[26,43,44,46]$, whereas the other three articles reported chatbots as an auxiliary component complementing other intervention approaches such as messages and conversations delivered by human facilitators $[41,42,45]$ (Multimedia Appendix 1). The reviewed chatbots were designed with different theoretical components and varied in their abilities to engage in natural language conversations, relationship building, and emotional understanding. Overall, owing to a lack of reporting on the details of the theoretical framework and a limited number of RCT evaluations, it is difficult to systematically evaluate how different design theories and factors contribute to intervention efficacy. Based on this preliminary review, we identified a lack of systematic thinking in the development of AI chatbots for lifestyle behavior changes.

None of the studies reported in detail how they developed the chatbot program and none discussed ethical considerations regarding issues such as transparency, privacy, and potential algorithmic biases. Consequently, it remains unclear how to evaluate a chatbot's efficacy, the theoretical mechanisms through which chatbot conversations influence users, and potential ethical problems. To address these gaps, in the next section, we present our theoretical framework that delineates design considerations, core theoretical components supporting a chatbot's conversational capacity, multiple dimensions for usability and outcome evaluations, and ethical principles that need to be emphasized to guide development in this emerging field. 


\section{Results}

\section{AI Chatbots as Persuasive Technology}

We conceptualize behavior change chatbots as a type of persuasive technology [14], which is more complicated than designing a social chatbot to engage in general conversations (eg, talking about movies or weather) [47]. Persuasive technology broadly refers to computer systems that are designed to change the attitudes and behaviors of users [48]. Behavior change chatbots thus aim to change users' specific behaviors through engaging in conversations and delivering information and persuasive messages. In this regard, we propose that the chatbot dialog system needs to encompass two core capacities, including the relational capacity to establish and maintain a professional relationship with the user and the persuasive conversational capacity to change behaviors. Below, we describe a theoretical framework that elaborates on these two capacities and guides the design of AI chatbots for promoting physical activity and a healthy diet.

\section{Theoretical Framework: The AI Chatbot Behavior Change Model}

Figure 1 shows the theoretical framework for improving physical activity and diet using AI chatbots. We named this framework the AI chatbot behavior change model, which includes the following four major components: (1) designing chatbot characteristics and understanding user backgrounds; (2) building relational capacity; (3) building persuasive conversational capacity; and (4) evaluating mechanisms and outcomes. The four high-level components are specified in sequence to guide the design and evaluation of chatbots. This proposed model is based on reviewing relevant chatbot studies, recent developments in human-AI communication research, and innovations in NLP, as well as our own clinical and research experience and findings [23,49-54].

Figure 1. The artificial intelligence chatbot behavior change model.

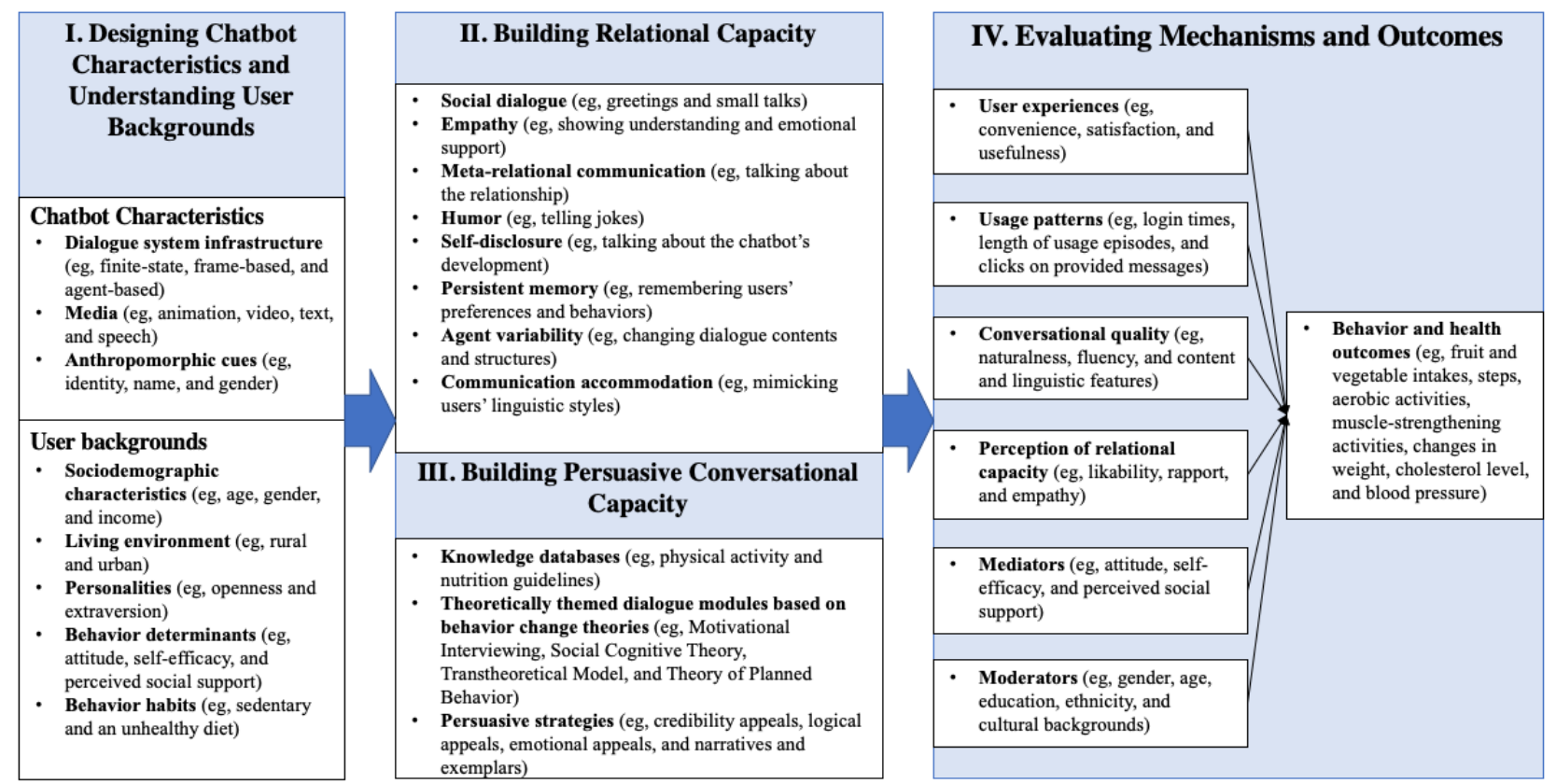

\section{Designing Chatbot Characteristics and Understanding User Background}

Chatbots are set up to mimic the characteristics of human-human conversations. Designing a chatbot requires both system-related and agent-related considerations. Upon choosing a system infrastructure (eg, finite-state, frame-based, and agent-based infrastructure) and media (eg, animation, video, text, and speech), the characteristics of a chatbot (eg, identity, name, and gender) can be specified. In the past, researchers have experimented with using a robot [32], animal [55], or human identity, ranging in degrees of applying anthropomorphic cues [56].

The computers are social actors (CASA) paradigm [57] and the uncanny valley effect (UVE) [58,59] are the most widely used theoretical frameworks for studying human-computer interactions. While the CASA paradigm assumes that humans can develop positive social relations with computer systems as the human familiarity of the system increases, the UVE argues that too much human familiarity would bring feelings of eeriness and discomfort. To increase a chatbot's social presence, some studies framed chatbots as peers and gave them gendered names (eg, Anna for female [27]). Deciding what name to call the chatbot and whether to frame it as a human peer or as a transparent bot system requires careful consideration. Our recent work [52] suggests that as AI chatbots are quickly adopting human conversational capacities, the perceived identity of a chatbot has significant effects on the persuasion outcome and interpersonal perceptions. Furthermore, our study findings suggest that users respond better if the chatbot's identity is clearly presented. This may be because users can develop more agency and control if they know how to respond to the 
conversational partner by applying different communication norms. For instance, if a chatbot is presented with a human identity and tries to imitate human inquiries by asking personal questions, the UVE can be elicited and make people feel uncomfortable [52]. However, contrary findings have also been identified as some studies show evidence that people respond well and disclose more personal information if the chatbot is presented as a bot and can also display emotions [60,61]. Identifying the boundary conditions for chatbot identity and disclosures in various application contexts requires more research to provide empirical findings.

Designing a personalized chatbot system requires the understanding of each individual user's background (eg, sociodemographic characteristics, living environment, and personality), behavior determinants, and habits [62-65]. The assumption is that a personalized intervention is more effective as it tailors both behavior change strategies and persuasive messaging to each user's unique background and needs to achieve personally optimized outcomes [63]. In general, the first component serves to set up the chatbot characteristics and collect useful user background information to inform the development of algorithms supporting the second component and the third component. Theoretically, user background information can be incorporated as contextual information to develop algorithms to generate personalized relational messages and persuasive messages. Which characteristics can be used to tailor which messages depends largely on the target population's needs and preferences $[66,67]$. Past literature has examined a number of useful characteristics for personalized influences, such as using different persuasive strategies to appeal to different personality traits $[53,68]$ or setting personalized change goals based on behavior habits [42]. In the realm of physical activity chatbot interventions, the Ally chatbot system by Kramer et al was able to welcome each participant using personalized messages and track individual physical activity using the smartphone's built-in accelerometer [42]. The system specifically set a personalized activity goal slightly above the participant's current average activity level. Along this line, the application of control systems engineering in modeling individuals' behavior states and adapting personalized goals over time is a promising approach [22].

\section{Building Relational Capacity}

In order to use an AI chatbot as a social conversational agent, we emphasize designing the system's relational capacity in chatbot and user interactions [29,69-72]. Bickmore et al provided extensive discussions on the principles of building relational capacity in behavior change agents, such as using social dialog, empathy, meta-relational communication (talk about the relationship), humor, self-disclosure, persistent memory, and agent variability [70]. One of their studies showed that when compared to a nonrelational agent, a relational agent was more respected, liked, and trusted, which led to more positive behavior changes [29].

It is worth noting that most of the reported relational agents are embodied virtual agents, taking on specific anthropomorphic cues and nonverbal behaviors but using restricted scripted dialog designs. It remains less clear what relational capacity a nonembodied chatbot can achieve just through natural language conversations. Recent endeavors to accelerate natural conversations in everyday social companion chatbots have yielded promising results. One study reported that users of a companion chatbot (called "Replika") perceived the chatbot to be human-like, intelligent, supportive, and able to facilitate social connection. However, UVEs also emerged as some users felt that the chatbot's conversation was too natural and thus "creepy" [73]. In another case study that analyzed user reviews of the Amazon chatbot device, researchers found that over half of the reviewers referred to the chatbot using the personified name "Alexa," and as users' social interactions with the device increased, a greater level of personification occurred, which was associated with increased product satisfaction [74]. This suggests that people tend to personify Alexa, which is in line with the CASA paradigm. As a chatbot's natural conversational abilities continue to rapidly improve, it is likely that relational capacity building can lead to better user engagement and retainment, despite other technological limitations.

To scale up the relational capacity in chatbots, conversational norms and relational strategies need to be built into the system. One approach can be through extracting patterns from longitudinal human-human conversations and drawing on theories from interpersonal communication and the latest human-AI communication research $[75,76]$. For example, the integrated model of advice giving [77,78] and the communication accommodation theory $[79,80]$, combined with the chatbot's capacity of persistent memory (eg, storing conversation history) and variability (eg, changing conversation content and structure), can provide useful insights in guiding the structure of conversations and specific choices in linguistic, semantic, and sentence styles.

\section{Building Persuasive Conversational Capacity}

Programs delivered by chatbots need to possess the core knowledge structures and intervention messages used in traditional approaches. Building behavior change messages into chatbot conversations first requires curating knowledge databases regarding physical activity and dietary guidelines. Thereafter, relevant behavior change theories need to be applied to generate themed dialog modules (eg, goal setting, motivating, and proving social support). Commonly used behavior change theories include motivational interviewing [81], the social cognitive theory [56], the transtheoretical model [82], and the theory of planned behavior [83]. One approach is to design human-human conversation episodes based on addressing each of the theoretical concepts (eg, a human interventionist providing social support to a participant) and to develop dialog modules that mimic such conversations.

In addition to delivering theory-based intervention messages, chatbots' efficacy in eliciting behavior changes can be augmented by employing persuasive messaging strategies [84]. This thinking stems from the line of work in public health communication that aims to integrate behavior change theories and message effect theories (ie, theories that direct the selection of specific persuasive appeals and message features to enhance the effectiveness of communication) [85]. Persuasive strategies are designed to motivate behavior changes and are nuanced 
messaging choices to enhance attention, trust, and engagement, or to influence cognitive and emotional reactions. Persuasive strategies are important in shaping, changing, and reinforcing people's attitudes and behaviors. Previous research has shown that even simply asking questions about a behavior can lead to changes in the behavior, known as the "question-behavior" effect. For instance, one study found that asking people questions about exercise led to an increase in self-reported exercise [86]. Although this effect was small and based on survey reports, it suggests that questions can function as a reminder or cue to action. Thus, one task of chatbots can be to ask questions to allow users to reflect and then get motivated for behavior change. More persuasive strategies can be embedded into theoretically themed dialog modules, such as using classical rhetorical appeals [53,68], including credibility appeals (eg, showing messages from sources that the target audiences trust), logical appeals (eg, providing reasoning and evidence for benefits of physical activity and a healthy diet), and emotional appeals (eg, using fear, guilt, or hope appeals for motivation). In addition, specific persuasive messaging strategies, such as using narratives and exemplars (eg, telling stories to enhance self-efficacy), can also enhance personal involvement and engagement. For example, to augment the approach of motivational interviewing, we can consider using credibility appeal to strengthen user's trust in the chatbot, so that they become more comfortable in disclosing thoughts. In addition, to augment the approach of social cognitive theory, we can consider constructing narrative exemplars in terms of talking about relevant peers' successful experiences to boost participants' self-efficacy.

One common limitation of traditional programs is the static nature of persuasive messages, because of infrequent measurements of behaviors and users' behavior change stages. Chatbots deployed on smartphones can address this limitation by utilizing ecological momentary assessment methods, in-built accelerometers, GPS, and other sensors, in addition to collecting user-reported data from convenient short surveys through the smartphone. For instance, research has shown that an accelerometer installed on smartphones is accurate for tracking step count [9] and that GPS signals can be used to estimate activity levels [87]. By objectively tracking and modeling activity patterns, developing machine learning models to update personalized goals and persuasive messages becomes feasible. Our work has shown that by using steps and physical activity intensity records, models can predict an individual's probability of disengagement from the intervention [88]. Further, by using NLP and cluster analysis, we could differentiate individuals' motivation levels as communicated in the conversation to tailor intervention maintenance programs [23]. These results indicate that AI chatbots can adapt not only behavior change goals and techniques, but also conversational styles (eg, emotional tones) based on learning from a user's natural language inputs to enhance the engagement and effectiveness of messages.

Furthermore, rapid progress in mobile health technologies and functions has enabled the design of just-in-time adaptive interventions (JITAIs) [24]. JITAI designs in combination with real-time data from ecological momentary assessment, in-built accelerometers, GPS, and/or other sensors will allow chatbots to customize the timing, amount, content, and frequency of the intervention, by adapting each individual's internal and external changes over time. However, a recent scoping review of health care chatbots showed that the use of JITAIs in designing and evaluating chatbots in health care in general and promoting physical activity and a healthy diet in particular is sparse, suggesting that future research needs to consider using more of these adaptive approaches [89].

\section{Evaluating Mechanisms and Outcomes}

Figure 1 shows the proposed dimensions for evaluating AI chatbot programs, including user experiences, usage patterns, conversational quality, perception of relational capacity, mediators, moderators, and behavior outcomes. All dimensions can be considered to improve the chatbot design and to understand theoretical mechanisms for how chatbot programs change behaviors.

User experiences concern users' subjective evaluations of the overall interaction with the system. Many scales have been developed to assess a program's convenience, satisfaction, usefulness, helpfulness, etc [90]. Usage patterns document objectively logged data regarding users' interactions with the system, including records such as login times, length of usage episodes, and clicks on provided messages [91]. Conversational quality can be measured from users' subjective evaluation of the conversation's coherence, naturalness, and fluency. In addition, objective content and linguistic analyses of conversations can be used to assess specific dimensions of conversations such as the length of conversations and amount of information exchanged. Perception of relational capacity evaluates users' perception of the chatbot identity and its relational capacity. Some studies have assessed the extent to which users deem a chatbot as a friend and its likability, as well as its capacity to achieve rapport, relate to human emotions, and show empathy [92-94]. Mediators refer to factors that help to explain why and how chatbot interventions are effective in promoting physical activity and a healthy diet. Chatbots can lead people to change their perceptions of themselves (eg, attitude, self-efficacy, and perceived social support) and help people to shape and form new behavior choices and patterns. These intermediate changes are important to explain the mechanisms of chatbot interventions and to design more effective interventions in the future. Moderators often refer to user characteristics such as gender, age, education, ethnicity, and cultural backgrounds, and these subgroups (eg, men vs women) may respond to a chatbot intervention differently. Advances in digital technologies can unintentionally reinforce or increase existing health disparities [95]. Thus, evaluating moderation effects is crucial in documenting a potential digital divide or lack thereof. Lastly, behavior outcomes denote actual changes in behavior and health, including diet (eg, fruit and vegetable intake five times per day [96]) and physical activity changes (eg, daily steps, aerobic activities, and muscle-strengthening activities [97]), and subsequent effects on health outcomes such as weight and blood pressure.

\section{Ethical Considerations}

General ethical principles and guidelines for AI's integration in health care need to be adopted in designing chatbots for 
lifestyle modification programs [15,98-100]. Key ethical considerations include having transparency and user trust, protecting user privacy, and minimizing biases. To gain the trust of users, credibility and transparency have to be established and communicated. A brief introduction of the intention and expertise of the research team behind the chatbot may enhance its credibility. Similarly, providing users with high-level explanations on the machine learning algorithms and data processing can help increase transparency. Protection of user privacy faces multiple challenges. There is emerging research showing that multiple sets of anonymized data can be modeled to reidentify individuals $[101,102]$. In the context of chatbot interventions, high standards of confidentiality and data anonymization, such as differential privacy [103], need to be adopted to decrease the risks of reidentification.

Within the context of persuasive health technology, beyond considering the general ethical principles in AI described above, another central framework that needs to be incorporated is the bioethics framework [104] consisting of (1) nonmaleficence, (2) beneficence, (3) respect for autonomy, and (4) justice. Nonmaleficence means the obligation to not inflict any harm or incur the least harm possible to reach a beneficial outcome. Beneficence denotes a moral obligation to act for others' benefits. Building a commitment to nonmaleficence and beneficence means the chatbot's intent is to benefit users with information, knowledge, care, and guidance, as well as to take positive steps to prevent and remove harm from the user. For example, chatbots need to be designed to understand expressions from users that indicate they may be undergoing difficult situations requiring human moderators' help. Specifically, it is important to foresee and preemptively plan for the possibility that technical and algorithmic errors can occur, and it is pivotal to have human moderators in place to monitor user engagement regularly and be able to connect with users when challenging situations arise. Respect for autonomy means that the user has the capacity to act intentionally with understanding and without being controlled or manipulated by the chatbot. This specifies that users should be provided with full transparency about the intervention's goals, methods, and potential risks. Given the complexity in AI and technological designs, researchers need to strive to provide comprehensible explanations that users can understand and then take decisions for themselves [105]. In addition, users should be fully informed in the consent process and consent form as to how their data will be used to improve the chatbot overtime during or even after the intervention and should be given the opportunity to opt out of having their data used in this manner. Commitment to justice requires researchers to consider the technology's equity access and benefits to different populations, especially the consideration of high-needs users who are lower in socioeconomic status and digital literacy, or users with disabilities that could impact their interaction with chatbots. It is thus recommended that underserved populations, especially racial and ethnic minority groups, be represented and involved in all stages of the design and implementation of chatbot interventions to ensure health equity and social justice. Specifically, researchers need to consider applying debiasing strategies in building the dialog system [106,107] and socially aware algorithm design [108]. Given that the research field of using chatbots for behavior changes is still in its nascent phase, ensuring adherence to ethical principles and incorporating corresponding evaluative metrics is necessary for the field to move forward.

\section{Discussion}

In this paper, we reviewed and synthesized literature involving lifestyle modification program studies, theories and studies from behavior science and communication research, and technical advancements in AI and NLP, and proposed the AI chatbot behavior change model. The strength of the proposed model is that it considers a wide range of chatbot-related components, including chatbot/user characteristics, relational capacity, and persuasive conversational capacity, and points out potential mediating and moderating factors to be evaluated to establish the efficacy of chatbots in changing physical activity and diet behaviors, as well as health outcomes.

To our knowledge, this is the first theoretical framework to provide a guideline to design and evaluate chatbot-based physical activity and diet behavior interventions. We contextualize the framework in the domains of physical activity and diet behaviors because these two are frequent daily behaviors that need continued engagement and monitoring. Chatbots as a convenient conversational tool can connect with people in real time to optimize behavior change interventions.

Moving science forward, systematic approaches and interdisciplinary collaborations are needed to design effective AI-based chatbot physical activity and healthy eating programs. Our proposed theoretical framework is the first step to conceptualize the scope of the work and to synthesize all possible dimensions of chatbot features to inform intervention design. However, when applied in specific contexts, researchers and practitioners can prioritize certain features that are mostly relevant to the target population, according to initial formative research conducted with the target population [54]. In essence, we encourage researchers to select and design chatbot features through working with the target communities using stakeholder-inclusive and participatory design approaches $[109,110]$. We think such inclusive approaches are much needed and can be more effective in bringing benefits while minimizing unexpected inconvenience and potential harms to the community. In this regard, we do not mean that every new chatbot program has to be developed from scratch. Previously established effective programs and their highlighted features can be incorporated and translated to a chatbot program and pilot tested with the target population. From there, the above-mentioned JITAI approach can be studied to test how different features can be adaptively applied to different individuals over time.

In summary, our study calls for more interdisciplinary work to continue enriching the conceptualization of a chatbot as a relational and persuasive agent and to develop approaches to leverage AI techniques to improve a chatbot's relational and persuasive capacities with strong ethical principles. We call for future research to continue expanding and modifying this framework and to conduct empirical studies to evaluate its applicability in the actual design and assessment of interventions. 


\section{Acknowledgments}

This project was supported by a grant (K24NR015812) from the National Institute of Nursing Research; a grant (RAP Team Science Award) from the University of California, San Francisco; a grant (Artificial Intelligence in Healthcare Innovation Award) from the University of California, Davis; and a research gift from Intel. The study sponsors had no role in the study design; collection, analysis, or interpretation of data; writing of the report; or decision to submit the report for publication.

\section{Conflicts of Interest}

None declared.

\section{Multimedia Appendix 1}

Summary of chatbot-based physical activity and diet interventions. [DOCX File, $30 \mathrm{~KB}-$ Multimedia Appendix 1]

\section{References}

1. Cecchini M, Sassi F, Lauer JA, Lee YY, Guajardo-Barron V, Chisholm D. Tackling of unhealthy diets, physical inactivity, and obesity: health effects and cost-effectiveness. The Lancet 2010 Nov 20;376(9754):1775-1784. [doi:

10.1016/S0140-6736(10)61514-0] [Medline: 21074255]

2. Wagner K, Brath H. A global view on the development of non communicable diseases. Prev Med 2012 May;54 Suppl:S38-S41. [doi: 10.1016/j.ypmed.2011.11.012] [Medline: 22178469]

3. Bennett JE, Stevens GA, Mathers CD, Bonita R, Rehm J, Kruk ME, et al. NCD Countdown 2030: worldwide trends in non-communicable disease mortality and progress towards Sustainable Development Goal target 3.4. The Lancet 2018 Sep;392(10152):1072-1088. [doi: 10.1016/S0140-6736(18)31992-5]

4. Bloom DE, Cafiero ET, Jané-Llopis E, Abrahams-Gessel S, Bloom LR, Fathima S, et al. The Global Economic Burden of Noncommunicable Diseases. World Economic Forum. 2011 Sep. URL: http://www3.weforum.org/docs/ WEF Harvard HE GlobalEconomicBurdenNonCommunicableDiseases 2011.pdf [accessed 2020-08-30]

5. Clarke TC, Norris T, Schiller JS. Early Release of Selected Estimates Based on Data From the 2018 National Health Interview Survey. National Center for Health Statistics. 2019. URL: https://www.cdc.gov/nchs/nhis/releases/released201905. $\underline{\text { htm }}$ [accessed 2020-08-28]

6. Hales C, Carroll M, Fryar C, Ogden C. Prevalence of Obesity and Severe Obesity Among Adults: United States, $2017-2018$. NCHS Data Brief. 2020. URL: https://www.cdc.gov/nchs/data/databriefs/db360-h.pdf [accessed 2020-08-29]

7. Bertram M, Banatvala N, Kulikov A, Belausteguigoitia I, Sandoval R, Hennis A, et al. Using economic evidence to support policy decisions to fund interventions for non-communicable diseases. BMJ 2019 May 20;365:11648. [doi: 10.1136/bmj.11648] [Medline: 31110025]

8. Vandelanotte C, Müller AM, Short CE, Hingle M, Nathan N, Williams SL, et al. Past, Present, and Future of eHealth and mHealth Research to Improve Physical Activity and Dietary Behaviors. J Nutr Educ Behav 2016 Mar;48(3):219-228.e1. [doi: 10.1016/j.jneb.2015.12.006] [Medline: 26965100]

9. Case MA, Burwick HA, Volpp KG, Patel MS. Accuracy of smartphone applications and wearable devices for tracking physical activity data. JAMA 2015 Feb 10;313(6):625-626. [doi: 10.1001/jama.2014.17841] [Medline: 25668268]

10. Flores Mateo G, Granado-Font E, Ferré-Grau C, Montaña-Carreras X. Mobile Phone Apps to Promote Weight Loss and Increase Physical Activity: A Systematic Review and Meta-Analysis. J Med Internet Res 2015 Nov 10;17(11):e253 [FREE Full text] [doi: 10.2196/jmir.4836] [Medline: 26554314]

11. Zhang J, Brackbill D, Yang S, Becker J, Herbert N, Centola D. Support or competition? How online social networks increase physical activity: A randomized controlled trial. Prev Med Rep 2016 Dec;4:453-458 [FREE Full text] [doi: 10.1016/j.pmedr.2016.08.008] [Medline: 27617191]

12. Zhang J, Brackbill D, Yang S, Centola D. Efficacy and causal mechanism of an online social media intervention to increase physical activity: Results of a randomized controlled trial. Prev Med Rep 2015;2:651-657 [FREE Full text] [doi: 10.1016/j.pmedr.2015.08.005] [Medline: 26844132]

13. Zhang J, Calabrese C, Ding J, Liu M, Zhang B. Advantages and challenges in using mobile apps for field experiments: A systematic review and a case study. 2017 Sep 05;6(2):179-196. [doi: 10.1177/2050157917725550]

14. IJsselsteijn W, de Kort Y, Midden C, Eggen B, van den Hoven E. Persuasive technology for human well-being: setting the scene. In: IJsselsteijn WA, de Kort YA, Midden C, Eggen B, van den Hoven E, editors. Persuasive Technology. PERSUASIVE 2006. Lecture Notes in Computer Science. Berlin, Heidelberg: Springer; 2006:1-5.

15. Walsh C, Chaudhry B, Dua P, Goodman KW, Kaplan B, Kavuluru R, et al. Stigma, biomarkers, and algorithmic bias: recommendations for precision behavioral health with artificial intelligence. JAMIA Open 2020 Apr;3(1):9-15 [FREE Full text] [doi: 10.1093/jamiaopen/ooz054] [Medline: 32607482] 
16. Laranjo L, Dunn AG, Tong HL, Kocaballi AB, Chen J, Bashir R, et al. Conversational agents in healthcare: a systematic review. J Am Med Inform Assoc 2018 Sep 01;25(9):1248-1258 [FREE Full text] [doi: 10.1093/jamia/ocy072] [Medline: $\underline{30010941]}$

17. Adam M, Wessel M, Benlian A. AI-based chatbots in customer service and their effects on user compliance. Electron Markets 2020 Mar 17:1-19. [doi: 10.1007/s12525-020-00414-7]

18. Androutsopoulou A, Karacapilidis N, Loukis E, Charalabidis Y. Transforming the communication between citizens and government through AI-guided chatbots. Government Information Quarterly 2019 Apr;36(2):358-367. [doi: 10.1016/j.giq.2018.10.001]

19. Georgescu A. Chatbots for education - trends, benefits and challenges. 2018 Presented at: eLearning \& Software for Education; April 20, 2018; Bucharest p. 195-200. [doi: 10.12753/2066-026x-18-097]

20. Nadarzynski T, Miles O, Cowie A, Ridge D. Acceptability of artificial intelligence (AI)-led chatbot services in healthcare: A mixed-methods study. Digit Health 2019;5:2055207619871808. [doi: 10.1177/2055207619871808] [Medline: 31467682]

21. Miner AS, Laranjo L, Kocaballi AB. Chatbots in the fight against the COVID-19 pandemic. NPJ Digit Med 2020 May 4;3(1):65 [FREE Full text] [doi: 10.1038/s41746-020-0280-0] [Medline: 32377576]

22. Conroy DE, Hojjatinia S, Lagoa CM, Yang C, Lanza ST, Smyth JM. Personalized models of physical activity responses to text message micro-interventions: A proof-of-concept application of control systems engineering methods. Psychol Sport Exerc 2019 Mar;41:172-180 [FREE Full text] [doi: 10.1016/j.psychsport.2018.06.011] [Medline: 30853855]

23. Fukuoka Y, Lindgren TG, Mintz YD, Hooper J, Aswani A. Applying Natural Language Processing to Understand Motivational Profiles for Maintaining Physical Activity After a Mobile App and Accelerometer-Based Intervention: The mPED Randomized Controlled Trial. JMIR Mhealth Uhealth 2018 Jun 20;6(6):e10042 [FREE Full text] [doi: 10.2196/10042] [Medline: 29925491]

24. Hardeman W, Houghton J, Lane K, Jones A, Naughton F. A systematic review of just-in-time adaptive interventions (JITAIs) to promote physical activity. Int J Behav Nutr Phys Act 2019 Apr 03;16(1):31 [FREE Full text] [doi: 10.1186/s12966-019-0792-7] [Medline: $\underline{\text { 30943983] }}$

25. Gabrielli S, Marie K, Corte C. SLOWBot (Chatbot) lifestyle assistant. In: Proceedings of the 12th EAI International Conference on Pervasive Computing Technologies for Healthcare. 2018 Presented at: PervasiveHealth '18; May 24, 2018; New York, United States p. 367-370. [doi: 10.1145/3240925.3240953]

26. Kocielnik R, Xiao L, Avrahami D, Hsieh G. Reflection Companion. Proc. ACM Interact. Mob. Wearable Ubiquitous Technol 2018 Jul 05;2(2):1-26. [doi: 10.1145/3214273]

27. Kowatsch T, Volland D, Shih I, Rüegger D, Künzler F, Barata F, et al. Design and evaluation of a mobile chat app for the open source behavioral health intervention platform MobileCoach. In: Maedche A, vom Brocke J, Hevner A, editors. Designing the Digital Transformation. DESRIST 2017. Lecture Notes in Computer Science. Cham, Switzerland: Springer; 2017.

28. Stein N, Brooks K. A Fully Automated Conversational Artificial Intelligence for Weight Loss: Longitudinal Observational Study Among Overweight and Obese Adults. JMIR Diabetes 2017 Nov 01;2(2):e28 [FREE Full text] [doi: 10.2196/diabetes.8590] [Medline: 30291087]

29. Sillice MA, Morokoff PJ, Ferszt G, Bickmore T, Bock BC, Lantini R, et al. Using Relational Agents to Promote Exercise and Sun Protection: Assessment of Participants' Experiences With Two Interventions. J Med Internet Res 2018 Feb 07;20(2):e48 [FREE Full text] [doi: 10.2196/jmir.7640] [Medline: 29415873]

30. Pereira J, Díaz Ó. Using Health Chatbots for Behavior Change: A Mapping Study. J Med Syst 2019 Apr 04;43(5):135. [doi: 10.1007/s10916-019-1237-1] [Medline: $\underline{\text { 30949846] }}$

31. Brixey J, Hoegen R, Lan W, Rusow J, Singla K, Yin X, et al. SHIHbot: A Facebook chatbot for sexual health information on HIV/AIDS. In: Proceedings of the 18th Annual SIGdial Meeting on Discourse and Dialogue. 2017 Presented at: The 18th Annual SIGdial Meeting on Discourse and Dialogue; 2017; Saarbrücken, Germany p. 370-373. [doi: $10.18653 / \mathrm{v} 1 / \mathrm{w} 17-5544]$

32. Fitzpatrick KK, Darcy A, Vierhile M. Delivering Cognitive Behavior Therapy to Young Adults With Symptoms of Depression and Anxiety Using a Fully Automated Conversational Agent (Woebot): A Randomized Controlled Trial. JMIR Ment Health 2017 Jun 06;4(2):e19 [FREE Full text] [doi: 10.2196/mental.7785] [Medline: 28588005]

33. Kramer LL, Ter Stal S, Mulder BC, de Vet E, van Velsen L. Developing Embodied Conversational Agents for Coaching People in a Healthy Lifestyle: Scoping Review. J Med Internet Res 2020 Feb 06;22(2):e14058 [FREE Full text] [doi: 10.2196/14058] [Medline: $\underline{32022693]}$

34. Hudlicka E. Virtual training and coaching of health behavior: example from mindfulness meditation training. Patient Educ Couns 2013 Aug;92(2):160-166 [FREE Full text] [doi: 10.1016/j.pec.2013.05.007] [Medline: 23809167]

35. Lucas GM, Rizzo A, Gratch J, Scherer S, Stratou G, Boberg J, et al. Reporting Mental Health Symptoms: Breaking Down Barriers to Care with Virtual Human Interviewers. Front. Robot. AI 2017 Oct 12;4:1-9. [doi: 10.3389/frobt.2017.00051]

36. Philip P, Micoulaud-Franchi JA, Sagaspe P, Sevin ED, Olive J, Bioulac S, et al. Virtual human as a new diagnostic tool, a proof of concept study in the field of major depressive disorders. Sci Rep 2017 Feb 16;7(12):42656 [FREE Full text] [doi: 10.1038/srep42656] [Medline: 28205601] 
37. Devlin J, Chang M, Lee K, Toutanova K. BERT: Pre-training of Deep Bidirectional Transformers for Language Understanding. In: Proceedings of NAACL-HLT 2019. 2019 Presented at: The 17th Annual Conference of the North American Chapter of the Association for Computational Linguistics: Human Language Technologies (NAACL-HLT 2019); June 7, 2019; Minneapolis, Minnesota.

38. Radford A, Wu J, Child R, Luan D, Amodei D, Sutskever I. Language models are unsupervised multitask learners. OpenAI Blog. 2019. URL: https://d4mucfpksywv.cloudfront.net/better-language-models/ language_models_are_unsupervised_multitask_learners.pdf [accessed 2020-08-29]

39. Wu Q, Zhang Y, Li Y, Yu Z. Alternating roles dialog model with large-scale pre-trained language models. arXiv. 2019. URL: https://arxiv.org/abs/1910.03756 [accessed 2020-08-29]

40. Gu J, Wu Q, Wu C, Shi W, Yu Z. A tailored pre-training model for task-oriented dialog generation. arXiv. 2020. URL: https://arxiv.org/pdf/2004.13835.pdf [accessed 2020-08-28]

41. Kramer J, Künzler F, Mishra V, Presset B, Kotz D, Smith S, et al. Investigating Intervention Components and Exploring States of Receptivity for a Smartphone App to Promote Physical Activity: Protocol of a Microrandomized Trial. JMIR Res Protoc 2019 Jan 31;8(1):e11540 [FREE Full text] [doi: 10.2196/11540] [Medline: $\underline{\text { 30702430] }}$

42. Kramer J, Künzler F, Mishra V, Smith S, Kotz D, Scholz U, et al. Which Components of a Smartphone Walking App Help Users to Reach Personalized Step Goals? Results From an Optimization Trial. Ann Behav Med 2020 Jun 12;54(7):518-528 [FREE Full text] [doi: 10.1093/abm/kaaa002] [Medline: 32182353]

43. Piao M, Ryu H, Lee H, Kim J. Use of the Healthy Lifestyle Coaching Chatbot App to Promote Stair-Climbing Habits Among Office Workers: Exploratory Randomized Controlled Trial. JMIR Mhealth Uhealth 2020 May 19;8(5):e15085 [FREE Full text] [doi: 10.2196/15085] [Medline: 32427114]

44. Stephens TN, Joerin A, Rauws M, Werk LN. Feasibility of pediatric obesity and prediabetes treatment support through Tess, the AI behavioral coaching chatbot. Transl Behav Med 2019 May 16;9(3):440-447. [doi: 10.1093/tbm/ibz043] [Medline: 31094445]

45. Maher CA, Davis CR, Curtis RG, Short CE, Murphy KJ. A Physical Activity and Diet Program Delivered by Artificially Intelligent Virtual Health Coach: Proof-of-Concept Study. JMIR Mhealth Uhealth 2020 Jul 10;8(7):e17558 [FREE Full text] [doi: $\underline{10.2196 / 17558}$ ] [Medline: $\underline{\text { 32673246] }}$

46. Fadhil A, Wang Y, Reiterer H. Assistive Conversational Agent for Health Coaching: A Validation Study. Methods Inf Med 2019 Jun;58(1):9-23. [doi: 10.1055/s-0039-1688757] [Medline: 31117129]

47. Shum H, He X, Li D. From Eliza to XiaoIce: challenges and opportunities with social chatbots. Frontiers Inf Technol Electronic Eng 2018 Jan 8;19(1):10-26. [doi: 10.1631/fitee.1700826]

48. Fogg BJ. Persuasive Technology: Using Computers to Change What We Think and Do. San Francisco, CA: Morgan Kaufmann; 2002.

49. Fukuoka Y, Gay CL, Joiner KL, Vittinghoff E. A Novel Diabetes Prevention Intervention Using a Mobile App: A Randomized Controlled Trial With Overweight Adults at Risk. Am J Prev Med 2015 Aug;49(2):223-237 [FREE Full text] [doi: 10.1016/j.amepre.2015.01.003] [Medline: 26033349]

50. Fukuoka Y, Haskell W, Lin F, Vittinghoff E. Short- and Long-term Effects of a Mobile Phone App in Conjunction With Brief In-Person Counseling on Physical Activity Among Physically Inactive Women: The mPED Randomized Clinical Trial. JAMA Netw Open 2019 May 03;2(5):e194281 [FREE Full text] [doi: 10.1001/jamanetworkopen.2019.4281] [Medline: 31125101]

51. Fukuoka Y, Vittinghoff E, Hooper J. A weight loss intervention using a commercial mobile application in Latino Americans-Adelgaza Trial. Transl Behav Med 2018 Sep 08;8(5):714-723 [FREE Full text] [doi: 10.1093/tbm/ibx039] [Medline: 29474702]

52. Shi W, Wang X, Oh Y, Zhang J, Sahay S, Yu Z. Effects of persuasive dialogues: testing bot identities and inquiry strategies. In: Proceedings of the 2020 CHI Conference on Human Factors in Computing Systems. 2020 Presented at: The 2020 CHI Conference on Human Factors in Computing Systems; April 30, 2020; Oahu, Hawaii p. 1-13. [doi: 10.1145/3313831.3376843]

53. Wang X, Shi W, Kim R, Oh Y, Yang S, Zhang J. Persuasion for good: towards a personalized persuasive dialogue system for social good. In: Proceedings of the 57th Annual Meeting of the Association for Computational Linguistics. 2019 Presented at: Annual Meeting of the Association for Computational Linguistics; August 2, 2019; Florence, Italy p. 5635-5649. [doi: $10.18653 / \mathrm{v} 1 / \mathrm{p} 19-1566]$

54. Zhang J, Jemmott Iii JB. Mobile App-Based Small-Group Physical Activity Intervention for Young African American Women: a Pilot Randomized Controlled Trial. Prev Sci 2019 Aug;20(6):863-872. [doi: 10.1007/s11121-019-01006-4] [Medline: 30788692]

55. Oviatt S, Darves C, Coulston R. Toward adaptive conversational interfaces. ACM Trans Comput Hum Interact 2004 Sep;11(3):300-328. [doi: 10.1145/1017494.1017498]

56. Phillips E, Zhao X, Ullman D, Malle B. What is human-like?: decomposing robots' human-like appearance using the Anthropomorphic roBOT (ABOT) database. In: Proceedings of the 2018 ACM/IEEE International Conference on Human-Robot Interaction. 2018 Presented at: ACM/IEEE International Conference on Human-Robot Interaction; March 8, 2018; Chicago, Illinois p. 105-113. [doi: 10.1145/3171221.3171268] 
57. Reeves B, Nass C. The Media Equation: How People Treat Computers, Television, and New Media Like Real People and Places. Cambridge, UK: Cambridge University Press; 1996.

58. Mori M, MacDorman K, Kageki N. The Uncanny Valley [From the Field]. IEEE Robot. Automat. Mag 2012 Jun;19(2):98-100. [doi: 10.1109/MRA.2012.2192811]

59. Kätsyri J, Förger K, Mäkäräinen M, Takala T. A review of empirical evidence on different uncanny valley hypotheses: support for perceptual mismatch as one road to the valley of eeriness. Front Psychol 2015 Dec;6(12):390 [FREE Full text] [doi: 10.3389/fpsyg.2015.00390] [Medline: 25914661]

60. Ho A, Hancock J, Miner A. Psychological, Relational, and Emotional Effects of Self-Disclosure After Conversations With a Chatbot. J Commun 2018 Aug;68(4):712-733 [FREE Full text] [doi: 10.1093/joc/jqy026] [Medline: $\underline{30100620}$ ]

61. Lee Y, Yamashita N, Huang Y, Fu W. "I Hear You, I Feel You": Encouraging Deep Self-disclosure through a Chatbot. In: Proceedings of the 2020 CHI Conference on Human Factors in Computing Systems. 2020 Presented at: The 2020 CHI Conference on Human Factors in Computing Systems; April 30, 2020; Oahu, Hawaii p. 1-12. [doi: 10.1145/3313831.3376175]

62. Vandelanotte C, De Bourdeaudhuij I, Brug J. Two-year follow-up of sequential and simultaneous interactive computer-tailored interventions for increasing physical activity and decreasing fat intake. ann. behav. med 2007 Jun 21;33(2):213-219. [doi: 10.1007/Bf02879903] [Medline: $\underline{5637250]}$

63. Krebs P, Prochaska JO, Rossi JS. A meta-analysis of computer-tailored interventions for health behavior change. Prev Med 2010 Sep;51(3-4):214-221 [FREE Full text] [doi: 10.1016/j.ypmed.2010.06.004] [Medline: 20558196]

64. Kaptein M, Markopoulos P, de Ruyter B, Aarts E. Personalizing persuasive technologies: Explicit and implicit personalization using persuasion profiles. International Journal of Human-Computer Studies 2015 May;77:38-51. [doi: 10.1016/j.ijhcs.2015.01.004]

65. Sakai R, Van Peteghem S, van de Sande L, Banach P, Kaptein M. Personalized Persuasion in Ambient Intelligence: The APStairs System. In: Keyson DV, editor. Ambient Intelligence. AmI 2011. Lecture Notes in Computer Science. Berlin, Heidelberg: Springer; 2011:205-209.

66. Kreuter MW, Wray RJ. Tailored and targeted health communication: strategies for enhancing information relevance. Am J Health Behav 2003;27 Suppl 3:S227-S232. [doi: 10.5993/ajhb.27.1.s3.6] [Medline: 14672383]

67. Bull FC, Kreuter MW, Scharff DP. Effects of tailored, personalized and general health messages on physical activity. Patient Education and Counseling 1999 Feb;36(2):181-192. [doi: 10.1016/s0738-3991(98)00134-7] [Medline: 10223022]

68. Lukin S, Anand P, Walker M, Whittaker S. Argument strength is in the eye of the beholder: audience effects in persuasion. In: Proceedings of the 15th Conference of the European Chapter of the Association for Computational Linguistics. 2017 Presented at: The 15th Conference of the European Chapter of the Association for Computational Linguistics; 2017; Valencia, Spain. [doi: 10.18653/v1/e17-1070]

69. Velicer W, Redding CA, Blissmer B, Babbin SF, Paiva A, Bickmore T, et al. Using relational agents in tailored interventions for multiple risk factors: preliminary 12 month results. Ann Behav Med 2013 Mar;45:S180.

70. Bickmore TW, Caruso L, Clough-Gorr K, Heeren T. 'It's just like you talk to a friend' relational agents for older adults. Interacting with Computers 2005 Dec;17(6):711-735. [doi: 10.1016/j.intcom.2005.09.002]

71. Bickmore T, Schulman D, Yin L, Ring L. Reaching minority populations with relational agents. Ann Behav Med 2013;45:S60.

72. Bickmore T, Schulman D, Pfeifer L, Yin L. Relational agents for promoting intervention engagement with underserved populations. Ann Behav Med 2011;41(Symposium 30A):3097.

73. Ta V, Griffith C, Boatfield C, Wang X, Civitello M, Bader H, et al. User Experiences of Social Support From Companion Chatbots in Everyday Contexts: Thematic Analysis. J Med Internet Res 2020 Mar 06;22(3):e16235 [FREE Full text] [doi: 10.2196/16235] [Medline: 32141837]

74. Purington A, Taft J, Sannon S, Bazarova N, Taylor S. "Alexa is my new BFF": Social Roles, User Satisfaction, and Personification of the Amazon Echo. In: Proceedings of the 2017 CHI Conference Extended Abstracts on Human Factors in Computing Systems. 2017 Presented at: The CHI Conference Extended Abstracts on Human Factors in Computing Systems; 2017; Denver, Colorado p. 2853-2859. [doi: 10.1145/3027063.3053246]

75. Mou Y, Xu K. The media inequality: Comparing the initial human-human and human-AI social interactions. Computers in Human Behavior 2017 Jul;72:432-440. [doi: 10.1016/j.chb.2017.02.067]

76. Hancock J, Naaman M, Levy K. AI-mediated communication: definition, research agenda, and ethical considerations. J Comput Mediat Commun 2020;25(1):89-100. [doi: 10.1093/jcmc/zmz022]

77. Feng B. Testing an Integrated Model of Advice Giving in supportive interactions. Hum Commun Res 2009;35(1):115-129. [doi: 10.1111/j.1468-2958.2008.01340.x]

78. MacGeorge EL, Guntzviller LM, Hanasono LK, Feng B. Testing Advice Response Theory in Interactions With Friends. Communication Research 2013 Nov 12;43(2):211-231. [doi: 10.1177/0093650213510938]

79. Gallois C, Gasiorek J, Giles H, Soliz J. Communication Accommodation Theory: Integrations and New Framework Developments. In: Giles H, editor. Communication Accommodation Theory: Negotiating Personal Relationships and Social Identities across Contexts. Cambridge, UK: Cambridge University Press; 2016:192-210.

80. Pretorius M. Communication accommodation theory analysis of nurse-patient interaction: Implications for course design. Int J Appl Linguist 2017 Aug 24;28(1):71-85. [doi: 10.1111/ijal.12184]

81. Miller W, Rollnick S. Motivational interviewing: helping people change. New York, NY: Guilford Press; 2012. 
82. Prochaska JO, Velicer WF. The transtheoretical model of health behavior change. Am J Health Promot 1997 Mar 21;12(1):38-48. [doi: 10.4278/0890-1171-12.1.38] [Medline: 10170434]

83. Fishbein M, Ajzen I. Predicting and changing behavior: The reasoned action approach. New York, NY: Psychology Press; 2011.

84. Dillard J, Pfau M. The persuasion handbook: Developments in theory and practice. Thousand Oaks, CA: Sage Publications; 2002.

85. Cappella J. Integrating message effects and behavior change theories: organizing comments and unanswered questions. J Commun 2006;56:S265-SS79. [doi: 10.1111/j.1460-2466.2006.00293.x]

86. Williams P, Block LG, Fitzsimons GJ. Simply asking questions about health behaviors increases both healthy and unhealthy behaviors. Social Influence 2006 Jun;1(2):117-127. [doi: 10.1080/15534510600630850]

87. Ellis K, Godbole S, Marshall S, Lanckriet G, Staudenmayer J, Kerr J. Identifying Active Travel Behaviors in Challenging Environments Using GPS, Accelerometers, and Machine Learning Algorithms. Front Public Health 2014 Dec;2(12):36 [FREE Full text] [doi: 10.3389/fpubh.2014.00036] [Medline: 24795875]

88. Zhou M, Fukuoka Y, Goldberg K, Vittinghoff E, Aswani A. Applying machine learning to predict future adherence to physical activity programs. BMC Med Inform Decis Mak 2019 Aug 22;19(1):169 [FREE Full text] [doi: 10.1186/s12911-019-0890-0] [Medline: 31438926]

89. Abd-Alrazaq A, Safi Z, Alajlani M, Warren J, Househ M, Denecke K. Technical Metrics Used to Evaluate Health Care Chatbots: Scoping Review. J Med Internet Res 2020 Jun 05;22(6):e18301 [FREE Full text] [doi: 10.2196/18301] [Medline: 32442157]

90. Bangor A, Kortum PT, Miller JT. An Empirical Evaluation of the System Usability Scale. International Journal of Human-Computer Interaction 2008 Jul 30;24(6):574-594. [doi: 10.1080/10447310802205776]

91. Deng T, Kanthawala S, Meng J, Peng W, Kononova A, Hao Q, et al. Measuring smartphone usage and task switching with $\log$ tracking and self-reports. 2018 Apr 25;7(1):3-23. [doi: 10.1177/2050157918761491]

92. Liu B, Sundar SS. Should Machines Express Sympathy and Empathy? Experiments with a Health Advice Chatbot. Cyberpsychol Behav Soc Netw 2018 Oct;21(10):625-636. [doi: 10.1089/cyber.2018.0110] [Medline: 30334655]

93. Lopatovska I, Williams H. Personification of the Amazon Alexa: BFF or a mindless companion. 2018 Presented at: The 2018 Conference on Human Information Interaction \& Retrieval; March 15, 2018; New Brunswick, New Jersey p. $265-268$. [doi: $10.1145 / 3176349.3176868]$

94. Bickmore T, Cassell J. Social dialogue with embodied conversational agents. In: van Kuppevelt JC, Dybkjær L, Bernsen NO, editors. Advances in Natural Multimodal Dialogue Systems. Dordrecht, Netherlands: Springer; 2005:23-54.

95. Mackert M, Mabry-Flynn A, Champlin S, Donovan EE, Pounders K. Health Literacy and Health Information Technology Adoption: The Potential for a New Digital Divide. J Med Internet Res 2016 Oct 04;18(10):e264 [FREE Full text] [doi: 10.2196/jmir.6349] [Medline: 27702738]

96. Kim DJ, Holowaty EJ. Brief, validated survey instruments for the measurement of fruit and vegetable intakes in adults: a review. Preventive Medicine 2003 Apr;36(4):440-447. [doi: 10.1016/s0091-7435(02)00040-3]

97. Reilly JJ, Penpraze V, Hislop J, Davies G, Grant S, Paton JY. Objective measurement of physical activity and sedentary behaviour: review with new data. Arch Dis Child 2008 Jul;93(7):614-619. [doi: 10.1136/adc.2007.133272] [Medline: 18305072]

98. Torresen J. A Review of Future and Ethical Perspectives of Robotics and AI. Front. Robot. AI 2018 Jan 15;4:1-10. [doi: $10.3389 /$ frobt.2017.00075]

99. Floridi L, Cowls J, Beltrametti M, Chatila R, Chazerand P, Dignum V, et al. AI4People-An Ethical Framework for a Good AI Society: Opportunities, Risks, Principles, and Recommendations. Minds Mach (Dordr) 2018 Nov 26;28(4):689-707 [FREE Full text] [doi: 10.1007/s11023-018-9482-5] [Medline: 30930541]

100. Kizilcec R. How much information? Effects of transparency on trust in an algorithmic interface. In: Proceedings of the 2016 CHI Conference on Human Factors in Computing Systems. 2016 Presented at: The 2016 CHI Conference on Human Factors in Computing Systems; May 12, 2016; San Jose, CA p. 2390-2395. [doi: 10.1145/2858036.2858402]

101. Na L, Yang C, Lo C, Zhao F, Fukuoka Y, Aswani A. Feasibility of Reidentifying Individuals in Large National Physical Activity Data Sets From Which Protected Health Information Has Been Removed With Use of Machine Learning. JAMA Netw Open 2018 Dec 07;1(8):e186040 [FREE Full text] [doi: 10.1001/jamanetworkopen.2018.6040] [Medline: 30646312]

102. McCoy TH, Hughes MC. Preserving Patient Confidentiality as Data Grow: Implications of the Ability to Reidentify Physical Activity Data. JAMA Netw Open 2018 Dec 07;1(8):e186029 [FREE Full text] [doi: 10.1001/jamanetworkopen.2018.6029] [Medline: 30646303]

103. Dwork C, Roth A. The Algorithmic Foundations of Differential Privacy. FNT in Theoretical Computer Science 2014;9(3-4):211-407. [doi: 10.1561/0400000042]

104. Engelhardt H. The foundations of bioethics. New York, NY: Oxford University Press; 1996.

105. Smids J. The voluntariness of persuasive technology. In: Bang M, Ragnemalm EL, editors. Persuasive Technology. Design for Health and Safety. PERSUASIVE 2012. Lecture Notes in Computer Science. Berlin, Heidelberg: Springer; 2012:123-132.

106. Liu H, Dacon J, Fan W, Liu H, Liu Z, Tang J. Does gender matter? Towards fairness in dialogue systems. arXiv. 2020. URL: https://arxiv.org/abs/1910.10486 [accessed 2020-08-29] 
107. Henderson P, Sinha K, Angelard-Gontier N, Ke N, Fried G, Lowe R, et al. Ethical challenges in data-driven dialogue systems. In: Proceedings of the 2018 AAAI/ACM Conference on AI, Ethics, and Society. 2018 Presented at: The 2018 AAAI/ACM Conference on AI, Ethics, and Society; February 3, 2018; New Orleans, Louisiana p. 123-129. [doi: 10.1145/3278721.3278777]

108. Kearns M, Roth A. The ethical algorithm: the science of socially aware algorithm design. New York, NY: Oxford University Press; 2019.

109. Sjöberg C, Timpka T. Participatory design of information systems in health care. J Am Med Inform Assoc 1998 Mar 01;5(2):177-183 [FREE Full text] [doi: 10.1136/jamia.1998.0050177] [Medline: 9524350]

110. Clemensen J, Larsen SB, Kyng M, Kirkevold M. Participatory design in health sciences: Using cooperative experimental methods in developing health services and computer technology. Qual Health Res 2007 Jan;17(1):122-130. [doi: $\underline{10.1177 / 1049732306293664]}$ [Medline: 17170250]

\author{
Abbreviations \\ AI: artificial intelligence \\ BERT: Bidirectional Encoder Representations from Transformers \\ CASA: computers are social actors \\ GPT2: Generative Pre-Training-2 \\ JITAI: just-in-time adaptive intervention \\ NCD: noncommunicable disease \\ NLP: natural language processing \\ RCT: randomized controlled trial \\ UVE: uncanny valley effect
}

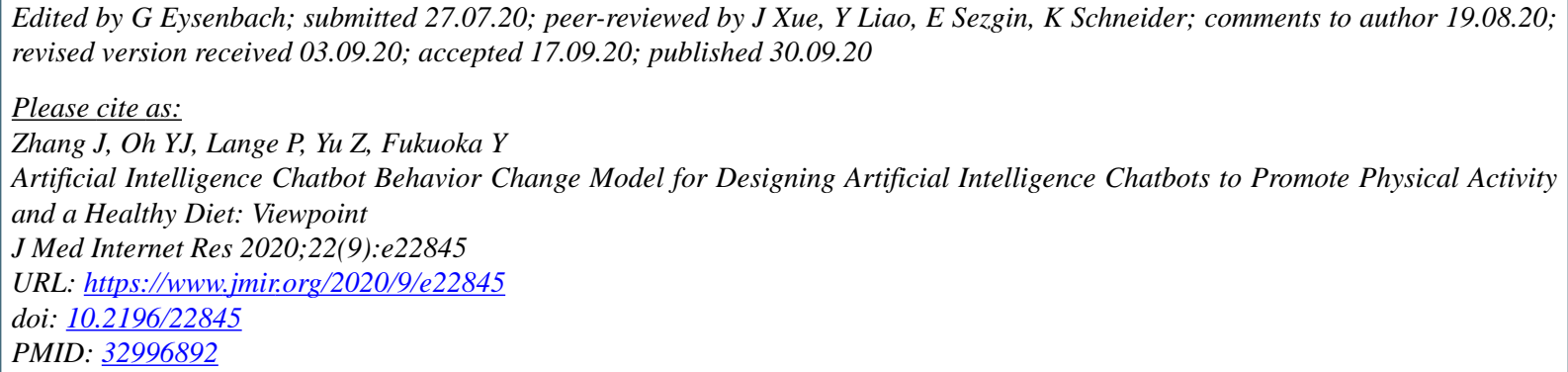

(CJingwen Zhang, Yoo Jung Oh, Patrick Lange, Zhou Yu, Yoshimi Fukuoka. Originally published in the Journal of Medical Internet Research (http://www.jmir.org), 30.09.2020. This is an open-access article distributed under the terms of the Creative Commons Attribution License (https://creativecommons.org/licenses/by/4.0/), which permits unrestricted use, distribution, and reproduction in any medium, provided the original work, first published in the Journal of Medical Internet Research, is properly cited. The complete bibliographic information, a link to the original publication on http://www.jmir.org/, as well as this copyright and license information must be included. 